\title{
Studies of three-body bound states with zero-range interaction in Minkowski space
}

\section{E Ydrefors*}

Instituto Tecnológico de Aeronáutica, DCTA, 12228-900 Sao Jose dos Campos, Brazil \& Université Paris-Saclay, CNRS/IN2P3, IJCLab, 91405 Orsay, France

E-mail: ydreforsakth. se

\section{J H Alvarenga Nogueira}

Dipartimento di Fisica, Universita di Roma La Sapienza \& INFN, Sezione di Roma Piazzale A.

Moro 5 - 00187 Roma, Italy

E-mail: dealvareeromal.infn.it

V A Karmanov

Lebedev Physical Institute, Leninsky Prospekt 53, 119991 Moscow, Russia

E-mail: karmanovva@ lebedev.ru

\section{T Frederico}

Instituto Tecnológico da Aeronáutica, DCTA, 12228-900 Sao Jose dos Campos, Brazil

E-mail: tobiaseita.br

The Bethe-Salpeter equation for three scalar bosons, with zero-range interaction, has been solved in Minkowski space by direct integration of the four-dimensional integral equation. The singularities appearing in the propagators were handled by standard analytical and numerical methods, without using any ansatz for the Bethe-Salpeter amplitude. Some of the results for the binding energies and transverse amplitudes are discussed here, and also compared with the corresponding results calculated in Euclidean space. A reasonable agreement between the calculations has been obtained.

Light Cone 2019 - QCD on the light cone: from hadrons to heavy ions - LC2019

16-20 September 2019

Ecole Polytechnique, Palaiseau, France

\footnotetext{
* Speaker.
} 


\section{Introduction}

The Bethe-Salpeter (BS) equation [1, 2] provides one framework for studies of relativistic few-body systems in the non-perturbative regime. From the numerical perspective, the easiest way to solve this integral equation is to perform its analytic continuation to the Euclidean space, by the Wick rotation [3]. Some physical quantities, e.g. binding energies, phase shifts and transverse amplitudes, are unchanged under this transformation. But, as discussed in Ref. [4], the Euclidean BS amplitude cannot be naively used to compute some dynamical observables such as electromagnetic form factors. For such applications one needs the BS amplitude solution in Minkowski space.

Understanding the properties of relativistic three-body systems is important for many applications within nuclear/particle physics, but it is more demanding compared to two-body systems. For this reason, many of the studies in the three-body context have, so far, been performed for the zero range interaction framework, which, although simple, is rather instructive. Investigations of the structure of three-body systems with short-range interactions are also of interest for Efimov physics which dominates the properties of the weakly-bound energy eigenstates with zero angular momentum, composed by the maximally symmetric configuration. But, it is well-known that in non-relativistic three-body systems with zero-range interaction the binding energy is unlimited from below, i.e. they undergo a so-called Thomas collapse [5]. This can be related to the Efimov effect and the scale invariance of the ultraviolet form of the non-relativistic equations. On the other hand, it was shown in Refs. [6, 7] that in a relativistic framework the Thomas collapse is not anymore equivalent to the Efimov effect, since the mass as a scale breaks the symmetry between the non-relativistic infrared region and the ultraviolet region. Furthermore, the Thomas collapse is eliminated by the appearance of an effective short-range repulsion of relativistic origin. Therefore, it is of great interest to perform studies of the structure of such systems within completely relativistic frameworks.

The BS and Light-Front (LF) equations for the three-scalar system with zero-range interaction were derived in Ref. [6]. The LF equation, which is deduced by integrating over $k^{-}$of the BS amplitude, or derived independently in the LF dynamics framework, only preserves the valence component of the BS amplitude, and was solved by Frederico in a restricted range and the solution was later generalized in Ref. [7]. Recently, in [8], we solved the BS equation, introduced in [6], in Euclidean space and it was then found that higher-Fock components beyond the valence have a huge impact on the structure of the three-body system. As already pointed out, it is crucial to acquire the BS amplitude directly in Minkowski space. Therefore, we solved in the recent work [9] the BS equation by direct integration in Minkowski space and some of the results are presented in this contribution. The results for the binding energies and transverse amplitudes are also compared with the ones obtained in Euclidean space.

\section{Three-body Bethe-Salpeter equation}

We consider here a system of three scalar bosons, with constituent masses $m$, with zero-range interaction. The BS equation for the Faddeev component of the vertex function is then given by [6]

$$
v(p, q)=2 i F\left(M_{12}^{2}\right) \int \frac{d^{4} k}{(2 \pi)^{4}} \frac{i}{\left[k^{2}-m^{2}+i \varepsilon\right]} \frac{i}{\left[(p-q-k)^{2}-m^{2}+i \varepsilon\right]} v(p, k),
$$


where $p$ represents the total four momentum of the three-body system and $q$ is the four momentum of the spectator particle. Furthermore, $F\left(M_{12}\right)$ is the two-body scattering amplitude and the squared mass of the two-body subsystem reads $M_{12}^{2}=(p-q)^{2}$.

The BS equation (2.1) constitutes a singular integral equation and is therefore cumbersome to solve numerically. If the goal only is to calculate well-defined quantities, e.g. binding energies, one can transform Eq. (2.1) to the complex plane through the Wick rotation. In the rest frame $(\vec{p}=\overrightarrow{0})$, the Euclidean BS equation obtains the form [8]

$$
v_{E}\left(q_{4}^{\prime}, q_{v}^{\prime}\right)=2 F\left(-M_{12}^{\prime 2}\right) \int_{-\infty}^{\infty} d k_{4}^{\prime} \int_{0}^{\infty} \frac{d k_{v}^{\prime}}{(2 \pi)^{3}} \frac{\Pi_{E}\left(q_{4}^{\prime}, q_{v}^{\prime}, k_{4}^{\prime}, k_{v}^{\prime}\right)}{\left(k_{4}^{\prime}-\frac{i}{3} M_{3}\right)^{2}+k_{v}^{\prime 2}+m^{2}} v_{E}\left(k_{4}^{\prime}, k_{v}^{\prime}\right)
$$

where the kernel $\Pi_{E}$ is obtained from the second propagator in (2.1) after Wick rotation and integration over the angles.

The three-body LF equation, derived in Ref. [6], is fully defined in Minkowski space, but it only gives access to the valence component of the BS amplitude. For more realistic computations of dynamical observables, it is thus important to also study the full solution of Eq. (2.1) directly in Minkowski space. Following the formalism presented in [10], we decompose the propagator $\left[k^{2}-m^{2}+i \varepsilon\right]^{-1}$ as

$$
\frac{1}{k^{2}-m^{2}+i \varepsilon}=P V \frac{1}{k_{0}^{2}-\varepsilon_{k}^{2}}-\frac{i \pi}{2 \varepsilon_{k}}\left[\delta\left(k_{0}-\varepsilon_{k}\right)+\delta\left(k_{0}+\varepsilon_{k}\right)\right]
$$

where $\varepsilon_{k}=\sqrt{k_{v}^{2}+m^{2}}$ and $k_{v}=|\vec{k}|$.

Eq. (2.1) can subsequently be transformed to the partially non-singular form [9]

$$
\begin{aligned}
v\left(q_{0}, q_{v}\right) & =\frac{F\left(M_{12}^{2}\right)}{(2 \pi)^{4}} \int_{0}^{\infty} k_{v}^{2} d k_{v}\left\{\frac{\pi i}{\varepsilon_{k}}\left[\Pi\left(q_{0}, q_{v} ; \varepsilon_{k}, k_{v}\right) v\left(\varepsilon_{k}, k_{v}\right)+\Pi\left(q_{0}, q_{v} ;-\varepsilon_{k}, k_{v}\right) v\left(-\varepsilon_{k}, k_{v}\right)\right]\right. \\
& -2 \int_{-\infty}^{0} d k_{0}\left[\frac{\Pi\left(q_{0}, q_{v} ; k_{0}, k_{v}\right) v\left(k_{0}, k_{v}\right)-\Pi\left(q_{0}, q_{v} ;-\varepsilon_{k}, k_{v}\right) v\left(-\varepsilon_{k}, k_{v}\right)}{k_{0}^{2}-\varepsilon_{k}^{2}}\right] \\
& \left.-2 \int_{0}^{\infty} d k_{0}\left[\frac{\Pi\left(q_{0}, q_{v} ; k_{0}, k_{v}\right) v\left(k_{0}, k_{v}\right)-\Pi\left(q_{0}, q ; \varepsilon_{k}, k_{v}\right) v\left(\varepsilon_{k}, k_{v}\right)}{k_{0}^{2}-\varepsilon_{k}^{2}}\right]\right\}
\end{aligned}
$$

where the propagator singularities have been removed by subtractions. The kernel $\Pi$ contains now only weak (logarithmic) singularities which will be taken into account numerically and is given explicitly in [9].

The Minkowski BS amplitude is obtained from the vertex function by multiplying the latter by three external propagators. However, it cannot be yet compared with the corresponding Euclidean one. But, one can introduce the transverse variable $\vec{k}_{\perp}=\left(k_{x}, k_{y}\right)$ and define the transverse Faddeev BS component as [9]

$$
L_{1}\left(\vec{k}_{1 \perp}, \vec{k}_{2 \perp}\right)=\int_{-\infty}^{\infty} \frac{-i v\left(k_{1}, p\right) d k_{10} d k_{1 z} d k_{20} d k_{20}}{\left(k_{1}^{2}-m^{2}+i \varepsilon\right)\left(k_{2}^{2}-m^{2}+i \varepsilon\right)\left(k_{3}^{2}-m^{2}+i \varepsilon\right)} .
$$

Then the corresponding integral over $k_{4}, k_{z}$, due to the validity of the Wick rotation [3], from the Euclidean BS amplitude must be the same. As shown in Ref. [9] one can transform this equation into a less singular form, similar to (2.4), which is more suitable for numerical calculations. 


\section{Results and discussion}

In Ref. [9], we solved (2.4) by adopting a bi-cubic spline basis for the vertex function $v(p, q)$. In Table 1 is displayed the calculated eigenvalue $\lambda$, which multiplies the right-hand side of the BS equation, for three different values of the two-body scattering length. In the computations we adopted additionally as input the three-body binding energy obtained by solving the Euclidean BS equation derived in [8]. In the table, an eigenvalue of $\lambda=1.0$ means that Minkowski and Euclidean solutions are consistent. It is seen that for all three cases that the real part of $\lambda$ is close to one. To obtain a stable numerical solution, we adopted in the Minkowski-space calculations cut-offs on the variables $q_{0}$ and $q_{v}$. Differently, in the solution of the Euclidean BS equation the full integration domain could be retained. This is a plausible explanation for the small imaginary parts and the error of the order of $0.1 \%$ in the real parts.

\begin{tabular}{lll}
\hline$a m$ & $B_{3} / m$ & $\lambda$ \\
\hline-1.280 & 0.006 & $0.999-0.054 i$ \\
-1.500 & 0.395 & $1.000+0.002 i$ \\
-1.705 & 1.001 & $0.997+0.106 i$ \\
\hline
\end{tabular}

Table 1: Eigenvalues of the three-body ground state for three scattering lengths, $a$, calculated by using the Euclidean three-body binding energies.

Moreover, in Fig. 1 we show the modulus of the contribution $L_{1}\left(k_{1 \perp}, k_{2 \perp}=0\right)$ to the transverse amplitude for $B_{3} / m=0.395$, calculated by using Eq. (2.5). The results are also compared with the corresponding Euclidean ones. It is visible in the figure that the results are in fair agreement with each other. As can be seen in Fig. 1 of Ref. [9], $v\left(q_{0}, q_{v}\right)$ with respect to $q_{0}$ is a non-smooth function. Despite of this, the computed transverse amplitude versus $k_{\perp}$ is rather smooth, which makes the comparison even more remarkable.

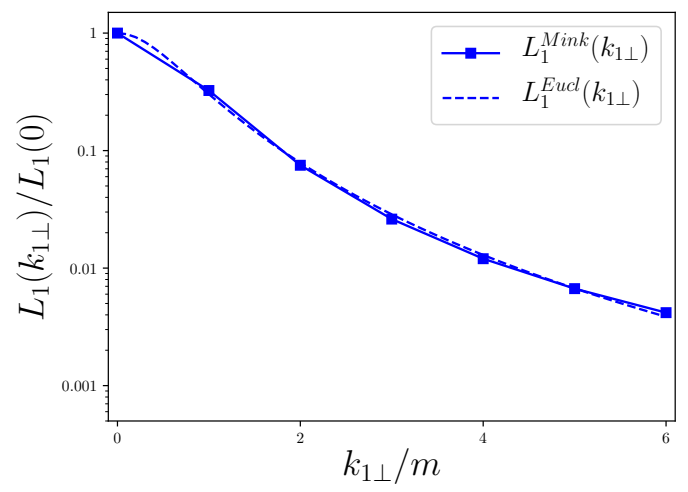

Figure 1: Transverse contribution, $L_{1}\left(k_{1 \perp}, k_{2 \perp}=0\right)$, obtained in Minkowski space compared with the one computed in Euclidean space, for the parameters $a m=-1.5$ and $B_{3} / m=0.395$.

\section{Conclusions}

We have in the present work, solved, directly in Minkowski space, the three-body BS equa- 
tion derived in [6] for scalar bosons interacting through the two-body zero-range interaction. The obtained results indicates that both the three-body binding energies and transverse amplitudes, derived by direct integration of the Minkowskian BSE, agree with the Euclidean ones. This should be the case because of the validity of the Wick rotation [3]. But, the method is rather demanding from the numerical perspective, since the appearance of many singularities which have to be treated properly. A possible path to improve the numerical accuracy and additionally be able to treat more realistic kernels and/or the spin degree of freedom is to transform the BS equation into a non-singular form by using the Nakanishi integral representation [11]. This work is in progress and calculations based on this method will be undertaken in the near future.

\section{Acknowledgments}

We are grateful to Jaume Carbonell for stimulating discussions. This study was financed in part by Conselho Nacional de Desenvolvimento Científico e Tecnológico (CNPq) and by Coordenação de Aperfeiçoamento de Pessoal de Nível Superior - Brasil (CAPES) - Finance code 001. J.H.A.N. acknowledges the support of the grants \#2014/19094-8 and \#2017/14695-1 and V.A.K. of the grant \#2015/22701-6 from Fundação de Amparo à Pesquisa do Estado de São Paulo (FAPESP). E.Y. thanks for the financial support of the grant \#2016/25143-7 and \#2018/21758-2 from FAPESP. We thank the FAPESP Thematic Projects grants \#13/26258-4 and \#17/05660-0. V.A.K. is also sincerely grateful to the group of theoretical nuclear physics of ITA, São José dos Campos, Brazil, for kind hospitality during his visits.

\section{References}

[1] E. E. Salpeter and H. A. Bethe, A Relativistic Equation for Bound-State Problems, Phys Rev 84 (1951) 1232.

[2] M. Gell-Mann and F. Low, Bound States in Quantum Field Theory, Phys Rev 84 (1951) 350.

[3] G. C. Wick, Properties of Bethe-Salpeter Wave Functions, Phys Rev 96 (1954) 1124.

[4] J. Carbonell and V. A. Karmanov, Solutions of the Bethe-Salpeter Equation in Minkowski Space and Applications to Electromagnetic Form Factors, Few-Body Syst 49 (2011) 205 [hep-ph/1012.0246].

[5] L. H. Thomas, The Interaction Between a Neutron and a Proton and the Structure of $H^{3}$, Phys Rev 47 (1935) 903.

[6] T. Frederico, Null-plane model of three bosons with zero-range interaction, Phys Lett B 282 (1992) 409.

[7] J. Carbonell and V. A. Karmanov, Three-boson relativistic bound states with zero-range two-body interaction, Phys Rev C 67 (2003) 037001 [nucl-th/0207073].

[8] E. Ydrefors et al, Three-body bound states with zero-range interaction in the Bethe-Salpeter approach, Phys Lett B 770 (2017) 131 [nucl-th/1703.07981].

[9] E. Ydrefors et al, Solving the three-body bound-state Bethe-Salpeter equation in Minkowski space, Phys Lett B 791 (2019) 276 [hep-ph/1903.01741].

[10] J. Carbonell and V. A. Karmanov, Solving Bethe-Salpeter scattering state equation in Minkowski space, Phys Rev D 90 (2014) 056002 [hep-ph/1408.3761].

[11] N. Nakanishi, Partial-Wave Bethe-Salpeter Equation, Phys Rev 130 (1963) 1230. 